\title{
Observations of magnetic fields toward the star forming region $\mathrm{S} 88 \mathrm{~B}$
}

\author{
Anuj P. Sarma ${ }^{1}$, C. L. Brogan ${ }^{2}$, T. Bourke ${ }^{3}$, and M. Eftimova ${ }^{1,4}$ \\ ${ }^{1}$ Physics Department, DePaul University, Chicago IL, USA \\ email: asarma@depaul.edu \\ ${ }^{2}$ National Radio Astronomy Observatory, Charlottesville, VA \\ ${ }^{3}$ Harvard-Smithsonian Center for Astrophysics, Cambridge, MA \\ ${ }^{4}$ Current Address: Dept. of Physics \& Astronomy, Univ. of North Carolina, Chapel Hill, NC
}

\begin{abstract}
We present observations of the high mass star forming region S88B taken with the VLA with the aim of measuring magnetic fields via the Zeeman effect. By observing thermal absorption lines of $\mathrm{OH}$ at 1665 and $1667 \mathrm{MHz}$, we obtain magnetic fields between 90 and 210 $\mu \mathrm{G}$. We find these magnetic fields to be dynamically significant in this region.
\end{abstract}

Keywords. H II regions - ISM: clouds - ISM: individual (S88B) - ISM: kinematics and dynamics - ISM: magnetic fields - ISM: molecules

\section{Introduction}

The importance of magnetic fields in the formation of stars has long been acknowledged. Despite remarkable advances in theory and instrumentation, however, observational data on magnetic fields is still scarce. Observations of the Zeeman effect in absorption lines with interferometers like the Very Large Array (VLA) provide an excellent method of mapping the magnetic field in regions that are along the line of sight toward strong background continuum sources (e.g., Brogan \& Troland 2001; Sarma et al. 2000). With these considerations in view, we have observed the star forming region S88B with the VLA for the Zeeman effect in thermal absorption lines of $\mathrm{OH}$ at 1665 and $1667 \mathrm{MHz}$.

\section{Observations \& Data Reduction}

The observations were carried out with the Very Large Array (VLA) of the NRAO in 2003 in the B-configuration, and combined with C-configuration data observed in 1997. Both right (RCP) and left (LCP) circular polarizations and both main lines (1665 and $1667 \mathrm{MHz}$ ) were observed simultaneously. The data were calibrated and imaged using standard procedures in the AIPS package (NRAO) while the magnetic fields were determined using routines in the MIRIAD software package (BIMA/CARMA).

\section{Results \& Discussion}

Magnetic field strengths were determined by fitting a numerical frequency derivative of the Stokes $\mathrm{I}=(\mathrm{RCP}+\mathrm{LCP}) / 2$ spectrum to the Stokes $\mathrm{V}=(\mathrm{RCP}-\mathrm{LCP}) / 2$ spectrum. The technique is described in detail in Roberts et al. (1993). The results of the fits give the line-of-sight component of the magnetic field, $B_{\text {los }}$. Figure 1 shows the resulting $B_{\text {los }}$ map (from the $1665 \mathrm{MHz}$ data for which the derived value of $B_{\text {los }}$ is greater than the $3 \sigma$ level). In the area enclosed by the ellipse in Fig. 1, the detected field is above the $3 \sigma$ 


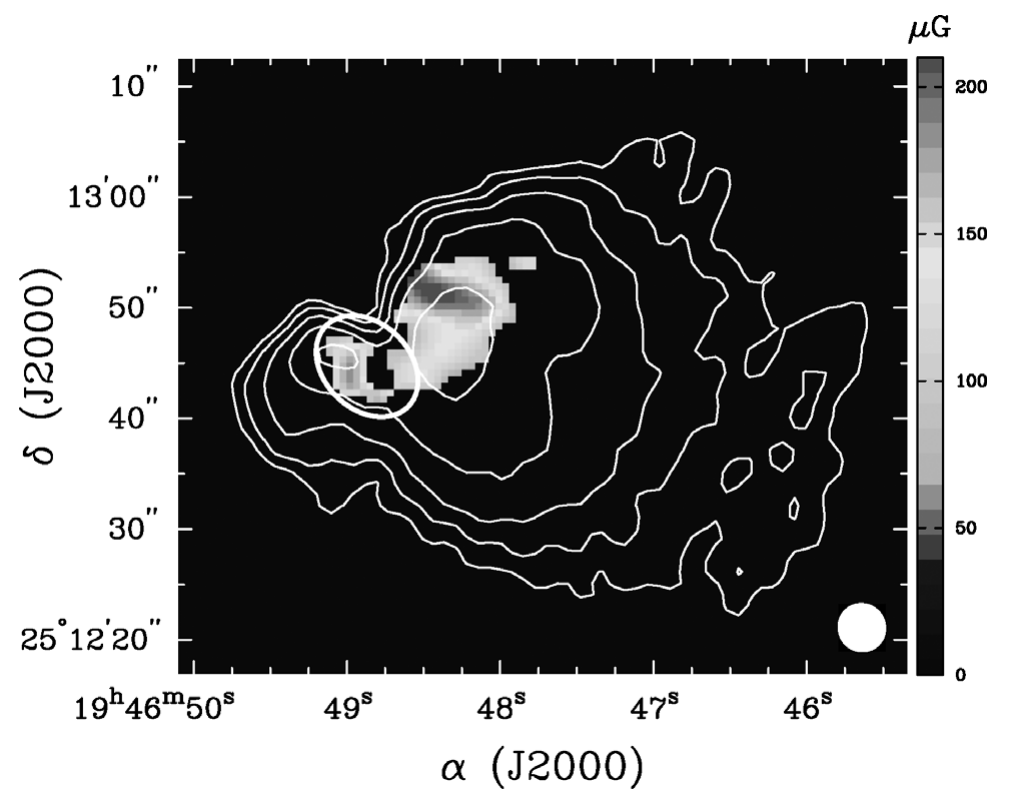

Figure 1. Gray-scale image of the detected $B_{\text {los }}$ toward S88B. The filled ellipse in the bottom right represents the beam for the $\mathrm{OH}$ absorption line observations $\left(4.5^{\prime \prime} \times 4.3^{\prime \prime}, \mathrm{PA}=14^{\circ}\right)$. The contours depict the $18 \mathrm{~cm}$ continuum, and are at $6,12,24,48,96 \mathrm{mJy}^{\text {beam }^{-1}}$.

level only in the $1665 \mathrm{MHz}$ line, whereas in the rest of the displayed $B_{\text {los }}$ map, the field is above the $3 \sigma$ level in both 1665 and $1667 \mathrm{MHz}$ lines.

In order to estimate of the importance of the magnetic field in a star forming cloud, we use the relation

$$
B_{S, \text { crit }}=5 \times 10^{-21} N_{p} \quad \mu \mathrm{G},
$$

where $N_{p}$ is the average proton column density in the cloud. The relation has been obtained by equating the static magnetic energy of the cloud to its gravitational energy, and $B_{S}$, crit is then the average static magnetic field in the cloud that would completely support it against self-gravity. The field can be judged to be dynamically important to the region even if it is less than $B_{S}$, crit, but is comparable to it. Using relevant physical parameters from our observations and the literature, we find $N_{p}=1.4 \times 10^{23} \mathrm{~cm}^{-2}$. The equation above then gives $B_{S}$, crit $=700 \mu \mathrm{G}$. Following Crutcher (1999), we use total (static) magnetic field strength equal to 2 times $B_{\text {los }}$. For our average adopted value for $B_{\text {los }}=150 \mu \mathrm{G}$ from Fig. 1, we find that the observed magnetic field is less than, but comparable to, the critical field. Therefore, the magnetic field should be dynamically significant, providing an important source of support against self gravity.

\section{Acknowledgements}

APS thanks the Chair of the Physics Department (Dr. Jesús Pando) and DePaul University for travel funds.

\section{References}

Brogan, C. L. \& Troland, T. H. 2001, ApJ 560, 821

Crutcher, R. M. 1999, ApJ 520, 706

Roberts, D. A., Crutcher, R. M., Troland, T. H., \& Goss, W. M. 1993, ApJ 412, 675

Sarma, A. P., Troland, T. H., Roberts, D. A., \& Crutcher, R. M. 2000, ApJ 533, 271 